\title{
Good Medical Practice for Drugs. Definition, Guidelines, References, Field of Action and Applications
}

\author{
The participants in Round Table $N^{\circ} 2$, Giens XXIII ${ }^{\star}$
}

Text received 8 July 2008, accepted 30 July 2008

\section{Keywords:}

drugs;

good medical practices; guidelines; proper use of drugs; references

\begin{abstract}
Proper use of drugs can be defined as the use of the right product, in a correct dosage, during an adequate length of time, for a given patient and provided he has no serious side effects.

It is virtually impossible, with such a number of drugs, such a number of clinical situations to prescribe adequately without using references or guidelines. References may lead to a unique choice, when the diagnosis is certain and the drug to be given is unique. With a good initial and continuous medical education, doctors can take easily this type of decision. The Summary of Products Characteristics (SPC) helps them; by sticking to this fundamental reference, prescription might be more precise and safe. In a lot of clinical situations the choice between a large numbers of therapeutic strategies necessitates use of a guideline based on scientific knowledge. Finally, a given therapeutic strategy can be as effective as and considerably less expensive than another. In such cases, payers can drive doctors to the prescription of the less expensive strategy.

Some difficulties are common to all references and guidelines:

1. A lot of clinical situations are not covered by guidelines.

2. Guidelines should be updated each time there is a modification of knowledge: it is extremely difficult to do.

3. A great number of guidelines exist, issued by scientific community, health authorities or the payers. Sometime you can find a proposition in a guideline and the reverse in another guideline. It could be confusing.

4. Guidelines should be evaluated rigorously to know if they fulfil their goals.

5. Some of those guidelines simply cannot help doctors. They are too complex or do not take into account practical situations. We have made an inventory of those various guidelines and their weaknesses and we propose some solutions to increase their utility. We propose an analysis of the situation and some solutions to improve the quality and the relevance of the guidelines: to create groups of coordination to choose the aims and the elaboration methods, to initiate a register of guidelines, to list the rules for any new guideline with quality control, to propose a continue medical education for all the health workers. It is necessary to measure the impact of any new recommendation and to do periodical actualisation. The goal of these recommendations is to improve global quality of health.
\end{abstract}

Proper use of drugs is a component of good medical practice. The simplistic definition is "usage of the right product, in a correct dosage, during an adequate length of time, for a given patient and provided he as no serious side effects".

Therapeutic result necessitates a prescription in accordance to rules, a correct delivery of drugs and that the patient takes effectively the treatment prescribed. In this paper, we will not consider anymore those aspects that were not really a topic of our work-

\footnotetext{
* For a list of participants, see the end of the article.
}

shop in Giens 2007. Our topic was clearly physicians' choice of drugs; what they refer to? Can they be guided by guidelines?

\section{From evidence based medicine to scientific guidelines}

With the multiplication of well-designed randomised controlled trials (RCT), a new paradigm has emerged in therapeutic "Therapeutic choice should be determined in reference to 
scientific data". But the model is more complex than this very simplistic description.

Therapeutic choice, for a given patient, prescribed by a given physician takes into account scientific data but also what the physician knows about this treatment and about the given patient and obviously what the patient desires.

This attempt at giving a scientific basis to the prescription is limited by the great number of data and the ability of a given physician to interpret them correctly. Moreover, a given patient is usually different, but from the patients included in RCT; older, younger, with renal or hepatic failure or receiving concomitant treatment not specifically evaluated in the primary file of the drug we intent to use.

It is, thus, necessary to integrate all the scientific data published and to stratify them as regards to the strength of evidence, to write a guideline aimed at helping other physicians.

Any scientific guideline must be objective and reliable. When the choice of a given drug is made, it is worthy to read the Summary of Product Characteristics (SPC) in order to make the more precise and secure prescription possible.

\section{When scientific guidelines are not necessary}

In current practice, prescription is made in reference to what the physician knows after a conscientious initial and continuous medical education. If the physician has any doubt on the best way to prescribe, he can find in the SPC of the drug, data to make a more precise prescription in term of dosage, duration, or special precaution in older patients or in patients with hepatic or renal failure. By reading the section of the SPC devoted to side effects and to drug-to-drug interactions, physician is able to prescribe more securely.

Simple prescription without the help of scientific guideline is very common. For example, a patient has a thyroid failure, and the cause has been determined; then substitutive treatment by thyroid hormones is easy to choose and easy to monitor with clinical symptoms and a single TSH (Thyroid Stimulating Hormone) blood test.

The experience of a given physician determines the number of clinical situations in which drug choice is almost automatic.

Another example is when an acute pulmonary oedema occurs in a patient with known congestive heart failure. Almost universally he will receive loop diuretics and subsequently initiation or adaptation of converting enzyme inhibitor. Knowledge of those elementary therapeutic choices must be conveyed by initial and continuous medical education more than by scientific guidelines.

This semi automatic medicine is limited by evolution of knowledge. In France during the sixties penicillin has been almost systematically given to children with angina and tonsillitis.
Inflammatory diseases, such as rheumatic fever or post streptococci nephritis, have disappeared. Then the concept of resistance to antibiotics has emerged and now the government has issued a campaign whose title is "antibiotics, they should not be automatic". Between 1960 and 2000, the goal of these treatments has radically changed: prevention of severe inflammatory diseases or prevention of the resistance to antibiotics.

Another example is the use of beta-blockers in heart failure; it used to be a tragic mistake, it is now an universal recommendation.

Again, adequate initial and continuous medical educations, evaluation of professional practices by comparison with those of other physicians lead to proper use of drugs in a daily practice. As it is simply impossible to know everything about a given drug, SPC must be easy to find to make prescription more precise and more secure.

\section{Guidelines taking into account economics data}

In France, budget allocated to drug expenses, is fixed by law each year. Similarly budget to cure a given pathology is fixed.

If a new drug appears or if indication for a given drug enlarges, new expenses occur. They have to be compensated by a close supervision of new prescriptions and a lowering of the costs of older treatments.

Supervision of prescription can be made by:

1. Authoritarian measures, such as suppression of reimbursement or promotion of generic drugs. Those measures have always a scientific basis, but are imposed to the physician.

2. Negotiated measures between users and payers. It is the case, in France, when health authorities and physicians' trade union sign an agreement on the proper use of some drugs. Similarly when a new drug is effective, is very expensive and should be given to a very limited number of patients, an agreement can be signed in order to increase the budget allocated to a given pathology.

\section{References and guidelines, how are they written?}

We have seen that guidelines can be written for various reasons. But it is always to help physicians. At the end of the day, physician can say my drugs' choice has been based on what I know from initial or continuous or medical education, or based on a given scientific guideline, or based on economical considerations. In every case, the physician has adapted his prescription to 
the given patient and by reading if necessary SPC or other documents issued by health authorities.

There are a large variety of objectives for writing a guideline: education, rationalisation, precision, and exploitation of all scientific data and or economic considerations. All those objectives are legitimate but influence the guidelines content.

\section{1. References for a given drug}

The initial step, for a drug, is the marketing authorisation. But now, in France, it must be followed by the description of how to lower or better estimate the risk of this drug. So the physician and the patient know which particular risk they have to anticipate and detect. Then, the transparency commission, in the High Authority for Health (HAS), issues an advice concerning, the opportunity to reimburse the product, its therapeutic benefit and to which patients it should be given. And finally, the Economic Committee for Health Products (CEPS), decides at what price the product will be reimbursed and what will be the yearly sell volume. Three steps and three opportunities for health authorities to ask for complementary studies: in patients underrepresented in the initial file (older or younger patients), to determine more accurately the benefit of the product in real life in France and finally to determine if the extra cost is compensated by a real clinical benefit.

All those advices are the basic data and the knowledge that a physician must have concerning the prescription of the new drug.

Changes of information concerning a product are necessary when new scientific knowledge appears. This evolution can be an extension or a restriction to the initial labelling of the drug.

Two opposite examples illustrate this process: Extension of labelling for herceptin in breast cancer and limitation of labelling for drugs used in hormonal treatment after menopause.

In those two cases, due to new scientific data, health authorities have mandated a group to update recommendations concerning a product or a class of products.

Very often, the physicians do not know changes about SPC. It is one of the major problems of the health authorities: How to communicate about changes of SPC. Changes can be minor, modest or sometimes important. They can be the result of a harmonisation at the European level and/or within a class of products. Those changes necessitate usually a great amount of job from pharmaceutical industry and health authorities and the process is very long.

Scientific news precede change of SPC and at a greater extent update of scientific guidelines.

Nevertheless SPC and other health authorities' advice are usually the basis of scientific guidelines.

\subsection{Scientific guidelines}

Writing a scientific guideline is a well-designed job.

First a small group of expert is in charge of putting together all the scientific information available. Then a writing committee try to integrate and balance those pieces of information to describe a therapeutic strategy for the clinical situation analysed. Finally a reading committee is in charge of evaluating the text and to introduce modifications necessary for fulfilling the goals of sufficiency, scientific exactitude and utility. This last parameter is of the utmost importance, as we will see later.

Usually guidelines are divided into two parts. The short one to which physician can refer in usual practice. It has to be as short as possible and driven by the purpose of describing the therapeutic strategies for the most common situations. The scientific basis is longer and allows finding which scientific data the authors are referring to.

\subsection{Guidelines taking into account economic data}

They are written in two main circumstances: when two various strategies have a similar clinical benefit and very different or when a very active and very expensive is marketed for a limited number of patients.

If a given hospital has an important recruitment of those patients, it is not fair to let it supports the cost excess. An agreement is then issued by which the cost of care for this disease is increases, but conversely physicians have to be able to demonstrate that patients treated clearly need this treatment.

\section{4. "Adequate use contract" for costly innovative new drugs}

In France, innovative and costly new drugs are reimbursed by the social security to the prescribing hospital on top of the reimbursement of the hospital stay. For these drugs and implantable devices, recommendations for a good clinical use (RBU) are elaborated by regional authorities and validated on a national basis by the Afssaps (French Health Products Safety Agency), the HAS or the INCa (National Institute for Cancer). Four categories are given:

I. Evident indications (authorised and on the market $=\mathrm{AMM}$ )

II. Accepted, relevant indications (on top of I situations, based on well conducted trials, clear evidences and favourable benefit/risk ratio).

III. Contraindicated, non-acceptable situations (with evidences for a negative benefit/risk ratio). 
IV. Other situations with lack of evidences for the evaluation of the benefit/risk ratio and/or no clear indication neither contraindication (few cases reports, small trials with a low level of evidences, compationable use...).

For these drugs usually used for inpatients, especially cancers, orphan diseases, severe immunological diseases, the prescriber has to give the precise indication leading to the prescription. Irrelevant prescriptions (Group III, some patients in group IV) could be not reimbursed to the user hospital.

\section{Some difficulties about writing and applying guidelines}

We have seen earlier problems encountered by health authorities to diffuse their recommendations on a given product. Those problems are even more important when the goal is to update and to verify utility of a scientific guideline.

\section{1. Which subject for a guideline?}

It is impossible to write a guideline for each clinical situation. We have said that for simple cases, knowledge is often sufficient. This is true too for disease in which treatment evolves rapidly or conversely for disease where treatment is almost ineffective.

The choice of a given topic depends also of the objectives of the authors. Physicians are now in face of numerous guidelines with various goals and sometimes contradictory evidences.

\subsection{Numerous guidelines and inequality of means}

When a scientific society decides to write a guideline, the goals and obligations are usually different than those of Health authorities.

The goal is to write a paper in a prestigious journal with the hope that this paper will be cited in a great number of papers afterwards. So periodically in United States, the Joint National Committee for Hypertension edits their recommendations for evaluation and treatment of high blood pressure. Similarly, the European Societies of Cardiology and Hypertension edits their recommendations. Journal editor who also wants the paper to be red and cited by a number of scientists helps them. Authors chosen by the scientific society, write their recommendations based on scientific data, without considering too closely SPC of products cited. Reasons for that are first that SPC may have not been updated and second that SPC are sometimes different from a country to another. A very special of guidelines can be edited by a scientific society when they are dedicated to few specialists of a given disease. For example guidelines for cancers treatment edited by INCa or guidelines to correct practice and indication of coronary angioplasty. In those last cases guidelines comprehension and applicability are not a major problem as guidelines are written by specialists chosen for their expertise and red by others specialists.

Conversely, guidelines edited by health authorities are usually concentrated on frequent or severe disease and dedicated to use of drugs widely prescribed. In those guidelines, scientific data are considered but content of SPC too. It is difficult for a given agency to edit contradictory recommendations in a SPC and in a guideline. In that guideline dedicated to the usual practice, the question of comprehension and applicability is extremely important. Guidelines are written by specialists but it is now the rule to include generalist practitioner in the writing and the reading committees; but despite this fact, guideline can be difficult to understand or to apply in the real life. The short part of the guideline must be dedicated to frequent clinical situations and submitted to an impact test involving users.

It should be stressed that it is less interesting for a high level scientist to write a guideline for health authorities than to make the same effort for a scientific society. Moreover, guidelines edited by health authorities rarely benefit from an editing process comparable to paper published in a prestigious journal.

\subsection{Updating guidelines}

Writing a guideline or a SPC is, as freezing an evolving picture. Scientific data, which modify what is known for a drug or for a class of drugs, are very slowly included in SPC, transparency commission's advice and guidelines edited by authorities.

This slow process is certainly justified for the marketing authorisation and admission to reimbursement. But it is a considerable obstacle for an adequate updating of guidelines or SPC. It can occur that SPC is outdated. An example is the use of ticlopidine and clopidogrel in the prevention of stent thrombosis. Current practice and scientific recommendations have recommended this use well before the change of the SPC.

Sometimes, important changes in economic data can induce a rapid evolution of recommendation. In United Kingdom, for example, use of statins was restricted up to the commercialisation of generics of simvastatin and pravastatin. Those products are now widely recommended and the use of statins is similar as it is in similar countries.

\subsection{Evaluating impact of guidelines}

Elaborating a guideline is very time-consuming and difficult. It should thus be natural to evaluate the impact of editing those 
texts. It is only possible if one can measure modification of therapeutic strategies and can evaluate the distance between ideal and practice. This work is very important as one can measure the same items at different point of time. When a guideline is widely accepted and applied, he is probably useful and easy to understand. If a part of the guideline is not applied and the remaining is accepted, there is probably a recommendation, which is far from clinical use in real life.

In some countries, it is easy to study cohorts of patients and to have data about diagnostic, treatment received and prognosis. Those data give information on the implementation of recommendation and to the difference of prognosis between patients treated in agreement with guidelines and those who are not.

In our country, we do not have access to such data. So we have to make specific studies evaluating, during a given length of time, adequacy of drug use in reference to guidelines. Those studies are often done by pharmaceutical industry with or without scientific instances. Again repetition of the same studies two to three years apart can measure the evolution of proper use of drugs.

Those various studies necessitate having access to diagnosis and treatment. In France we have not the tools to make such studies on a systematic basis. Computerized data are known by payers but they rarely use them to evaluate the impact of guidelines

\section{Improving current situation}

Choice of guidelines topics should be determined by a permanent observation of use of drugs. Priority should be given to frequent or costly diseases especially when clinical practice is heterogeneous. If a frequent off label use is detected reinforcement of recommendations and or advices for a better prescription is necessary. Rapid modification of recommendations should also be done when an important scientific event occurs.

Elaborating a guideline should essentially be done to help physicians and not only to gather all scientific information. The problem of multiple guidelines on the same topic seems to be difficult to solve. There should be coordination between health authorities and scientific societies to avoid too repetitive or too different guidelines. Some guidelines are edited periodically by scientific societies coming from all part of the world. It is the case of guidelines for use of antithrombotics. When this guideline is issued it seems futile to write another one involving the French having participated in the redaction of the first one. One could imagine that if a good scientific foreigner guideline exists, he could be used as a basis for a synthesis in French, even if health authorities want to add some data reflecting French specificities. Finally a guideline should not be edited before the realisation of an impact test on groups of generalist or specialist practitioners. They should give an advice about: Is it easy to read? Is it easy to understand? Do you think that this guideline will be useful in clinical practice?

Implementing a guideline necessitates first an adequate diffusion and next to elaborate a strategy for communicating this guideline. Pharmaceutical industry and payers have made opposite recommendation and the result is that physicians do what seems the best for a given patient without referring to guideline. It is clear that efficacy of guidelines would be greater if a minimal consensus between scientific societies, health authorities and even industry was obtained. It is important to make a standardized process for implementation of guidelines. Helping users should be the common goal. Usage of guidelines for authoritative measures is probably a mistake. It is a necessity to work on the best way for the prescriber to follow the guideline and the information has to be relay by various medical vehicles (patients association, non-medical press...). Helping tools for guidelines implementations are needed. Rules for the diffusion of the recommendations are to be the same for all the guidelines whatever the "origin" of the recommendations: pharmaceutical industry, colleges of physicians, payers... CME (continuous medical education) has to be a vector for the information and a way to follow its use. Physicians have to explain their choices when they prescribe out of the recommendations and the patient has to be informed. All these propositions are made to improve the adherence to the guidelines through the physicians, the health workers and the patients. Global diffusion rules might be useful.

Evaluating a guideline can be made with various tools, different methods and with opposite objectives. When evaluation is conducted a posteriori, by industry, scientists or payers, it can always be criticized. So evaluation has to be determined when guideline is published. Again a minimal agreement should be reached on tools that will be used for assessing guideline's efficacy. Similarly when the guideline is issued, it is necessary to build formation of physicians in order to improve acceptability of this guideline. The percentage of accordance to the recommendations might be a way to give credits to the physicians or accreditation for a health organisation. Post-marketing surveys are useful to improve the efficiency of the recommendations on the prescription decision.

Updating a guideline is very difficult and very slow. Nevertheless, with modern tools for communication, it should be possible to give information about results of complementary studies especially when authorities have requested them. Scientific information should also be completed more rapidly, especially when it modifies the use of a given product. Otherwise, physicians and scientists will not await health authorities' advice to modify their practice or their lecture. More generally a given guideline should not be considered as an adequate reference 5 to 7 years after it has been written. Update could be based on data coming from physicians, health authorities, or industry. The coordination could be 
made by HAS. Today this process is too slow due to conflicts of interest. This updating needs raging of the priorities, coordination, definition of priorities and pooling of the efforts of all the actors of health.

\section{Conclusion}

It is necessary that a unique authority be in charge of writing guideline when they appear to be necessary from the physicians' point of view or from the health authorities' point of view. In oncology, for example, recommendations issued by INCa have considerably changed clinical practices for the most frequent clinical situations. Thus a unique authority, taking into account existing guidelines and the actual clinical practice should be central for improvement of this work even if the sources of guidelines elaboration has to be multiple and complementary. A quality exigency is absolutely necessary. It should not be possible to edit a guideline without an adequate formation of users, or without a scientific project aimed at evaluating impact of the guideline on clinical practice and ultimately public health.

\section{Participants:}

Michel Andrejak (CHU, Amiens), Jean-Pierre Bader (Hôpital Fernand Vidal, Paris), Marion Bamberger (Bristol Myers Squibb), Jean-François Bergmann (Hôpital Lariboisière, Paris), Pascal Bilbault (Boehringer), Régis Bordet (CHU, Lille), Agnès Brouard (AGEPS), Anne Burstin (Afssaps), Alain Castaigne (AstraZeneca), Anne Castot (Afssaps), Alain Coulomb (Consultant, Paris), Muriel Dahan (Institut National du Cancer [INCa]), Frédéric DeBels (Haute Autorité de Santé [HAS]), Corinne Duguay (Sanofi Aventis), Nathalie Dumarcet (Afssaps), Pierre Fender (Caisse Nationale de l'Assurance Maladie [CNAM]), Christian Funck Brentano (Hôpital Saint Antoine, Paris); Isabelle Giri (Consultant, Verrières-le-Buisson), Pascale Jolliet (CHU, Nantes), Murielle Jousselon Pautrot (Afssaps), Florence Leclerc (Roche), Patricia Le Gonidec (Agence Régionale de l'Hospitalisation, Île de France [ARHIF]), Patricia Maillere (Iris),
François Meyer (Haute Autorité de Santé), Thierry Moreau Defarges (Janssen Cilag), Olivier Obrecht (HAS), Gilles Paintaud (CHU, Tours), Nicole Petitcollot (Direction Générale de la Santé [DGS]), Marc Samama (Hôpital Hôtel Dieu, Paris), Julia Sauterey (Observatoire des Médicaments des Dispositifs médicaux et des Innovations Thérapeutiques Île de France [OMEDIT IDF]), Pierre Schiavi (Servier), Philippe Tcheng (Sanofi Aventis), Patrick Villani (AP HM Hôpitaux de Marseille), Monique Weber (Caisse Nationale de l'Assurance Maladie des Travailleurs Salariés [CNAMTS]), Nadine Weisslinger Darmon (GlaxoSmithKline), Myriam Zylberman (Lilly).

\section{For more informations}

Décret n 2005-1023 du 24 août 2005 relatif au « contrat de bon usage des médicaments et des produits et prestations », mentionné à l'article L.162-22-7 du code de la sécurité sociale

Méthodologie générale d'élaboration des protocoles thérapeutiques «hors-GHS »: http://agmed. sante.gouv. fr/htm/3/t2a/html/methodologie.pdf

Accord de bon usage des soins relatif à l'utilisation des antiagrégants plaquettaires JO 5 janvier 2007

Accord-cadre national relatif au bon usage des antibiotiques dans les établissements de santé J.O n 150 du 29 juin 2002 page 11215 http://www. antibiotiquespasautomatiques.com/

Fiches de bon usage du médicament (BUM) HAS http://www .has-sante.fr/ portail/display.jsp?id=c_412202

Recommandations bonne pratique (RBP) Afssaps-HAS http://agmed.sante gouv. fr/htm/5/rbp/indrbp.htm

Les plans de gestion de risque http://agmed.sante.gouv.fr/htm/10/pgr/ indpgr.htm

Mise au point actualisée de juin 2006 du traitement hormonal substitutif de la ménopause. http://afssaps.sante.fr//htm/10/ths/thm_mise_ aupoint.pdf

Recommandations en hypertension artérielle et médecine cardiovasculaire http://www.sfhta.org/pages/recos_hta.html: 14 versions nationales et internationales (1997-2008)

Gusssous I, Rodondi N, Paccaud F. Accès sans prescription aux statines : bénéfique ou délétère ? Médecine de premier secours Revue médicale suisse 2005, 38: 2447-52

Comment améliorer la qualité de l'antibiothérapie dans les établissements de soins ? Qualité : préserver intérêt collectif sans nuire à l'intérêt individuel. SPILF. Presse Med 2002; 31 (26): 1231-39

Correspondence and offprints: Jean-François Bergmann, Service de Médecine Interne A, Hôpital Lariboisière, 2 rue Ambroise Paré, 75010 Paris, France. E-mail: jf.bergmann@lrb.aphp.fr 